\title{
Living Systems in Russia are Evaluated in Terms of the Number of Scientific Publications Related to the Subject
}

\author{
V.V.Sychev ${ }^{1 \#}$, A.V.Kolesnichenko ${ }^{1,2}$ \\ ${ }^{1}$ Park Media, Moscow \\ 2 Siberian Institute of Plant Physiology and Biochemistry of RAS \\ \# e-mail: vsychev@strf.ru
}

Those fields within science in which there is continuous development need to be objectively evaluated in order to determine the efficiency of work being conducted by those working in that field. Different criteria provide an estimate of this efficiency at both the level of individual scientists as well as that of entire scientific institutions. Moreover, it is possible to obtain an estimate of the rate of progress in research activities within different states.

he assessment of researchers and their respective level of activity is made based on the amount of research publications. This applies both to individual investigators and to scientific centers as a whole. There are some obvious advantages to this approach.

First, this is the most objective approach, because scientific publications are the most widespread and common measurable result of scientific work, being the vehicle by which research results can be distributed and shared with scientists all over the world.

Second, publication activity is an easily measured variable, especially in recent years, following the advent of new, large databases of research papers. It should be noted that different measurement systems register different scientific publications and, as a result, provide slightly different data. However, significant differences in figures and dynamics have not been observed in Russia.

With the aforementioned in mind, the publication activity (PA) of Rus- sian researchers involved in the "Living Systems" priority direction during the time period between 1996 and 2008 was analyzed using the international SCOPUS database. The "Living Systems" direction is second only to nanotechnologies in terms of the amount of financing but is first from the standpoint of resulting improvement in the quality of life in our country. The purpose of this analysis was to identify those specific spheres of the "Living Systems" Program where scientists are most active. The investigation consisted not only in the analysis of the number of publications, but also in the establishment of leading institutes and scientific centers able to become special "crystallization centers" with their work centered on "Living Systems" in Russia.

\section{METHOD}

Information from the SCOPUS database was used for this investigation. SCOPUS (www.scopus.com) is the largest abstract database in 
the world, containing more than 15,000 names of scientific, technical and medical publications from some 4,000 international publishing houses. Currently, the SCOPUS database includes more than 300 Russian academic periodicals.

Not all the fields of science related to living organisms were subject to analysis, but rather only those whose results could be used in actual practice. Choosing the particular fields to be included did not cause any difficulties: we analyzed the priority directions in science, technology and engineering development in the Russian Federation approved by the President in May of 2006. The priority directions include:

- Security and counter-terrorism

- Living systems

- Industry of nano-systems and nanomaterials

- Information and telecommunication systems

- Strategic weapons, military and special equipment

- Environmental conservation

- Transportation, aviation, and space systems

- Energy production and conservation

On May 21, 2006, the President approved a list of 34 technologies which were deemed critical for the Russian Federation; some of them are referred to in the "Living Systems" section. The list of critical technologies of the Russian Federation in the sphere of "Living Systems" includes the following critical technologies analyzed herein:

- Bio-information technologies

- Bio-catalytic, biosynthetic, and biosensor technologies
- Biomedical and veterinary technologies related to human and animal life support

- Genomics and post-genome drug formulation

- Cellular technologies

- Bioengineering technologies

- Biocompatible material technologies

Hence, these seven critical technologies were chosen for the present investigation. Using the previously determined set of key words for the search, we calculated the total number of articles published annually by Russian authors in either Russian or foreign scientific periodicals included in the SCOPUS database, separately for each of the above-mentioned categories of technologies. Each list of key words was always accompanied by the "Russia" affiliation.

It should be noted that many works are performed not only by one particular scientific team, but also within the framework of interdisciplinary projects realized by scientific consortiums comprising different institutes. Those data were also taken into account in the course of investigation.

Considering biology publications in the world in 1996-2008, the following picture emerges (Figs. 1a, 1b).

By far, the country with the greatest publication activity is the USA, and its PA increased during that period of time from 192,000 to 251,000 articles per year.

Among European countries, the most active in the sphere of science (Great Britain, Germany, France, and Italy) also demonstrated this trend. However, Great Britain and Germany have experienced a downturn in their PA during the last 2 to 3 years. Italy managed the greatest relative increase in the number of publications: in 12 years, the number of publications written by Italian scientists increased 1.6 times and reached a total of 35,000 articles a year. Switzerland demonstrated a non-significant increase in the number of biologyrelated articles (from 8.8 to 13.7 thousand).

Special attention should be paid to the increase in the number of articles published by scientists from the BRIC countries (Brazil, India, Russia, and China), which are referred to by experts as countries with growing economies. However, from the standpoint of scientific development, it is more reasonable to speak about a group composed of only three countries, with Russia being excluded from this group.

If, in 1996, Russia, China, and Brazil had almost equal starting positions $(6,400,6,200$ and 5,000 articles, respectively), the situation changed dramatically afterwards, primarily as a result of an unprecedented increase in the number of articles written by Chinese authors (57,400 articles in 2008). Currently, China is fourth in the world in the number of biological articles, surpassing even Germany, while Brazilian scientists published 19,000 articles in 2008. Over a period of years, India increased its PA from 11,000 articles in 1996 to 26,000 articles in 2008 , and it climbed to the eight position in the list of all countries. Although initially insignificant, the increase in the last few years experienced by Brazil has been remarkable, and it has sur-
Fig. 1 Russia and the World Leaders in the Sphere of Sciences Related to Living Organisms a) Total number of publications in biology in 19962008

b) Total number of publications in biology in 1996-2008 (USA excluded)

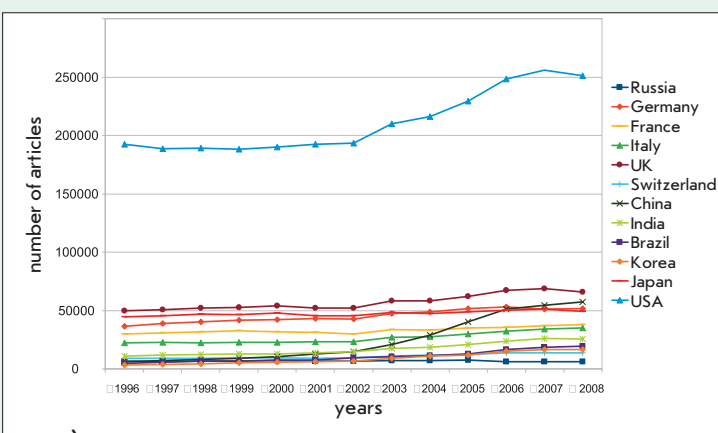

a)

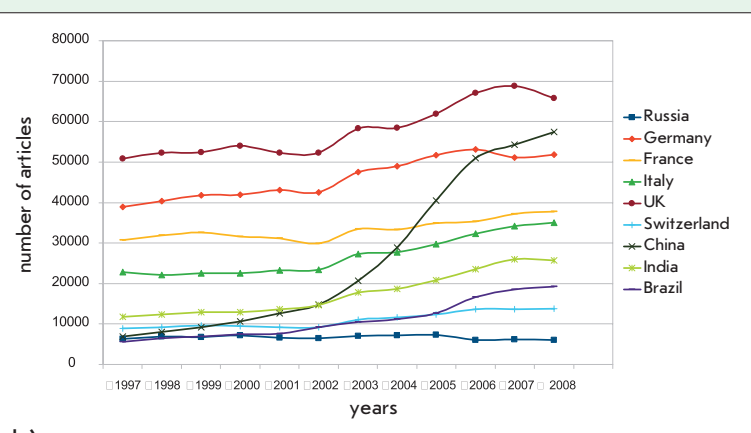

b) 
Fig. 2. Number of articles written by Russian researchers on bio-information technology.

\section{Fig. 3. Relationship} between the number of Russian articles on bio-information technology published in Russian journals versus in international journals.
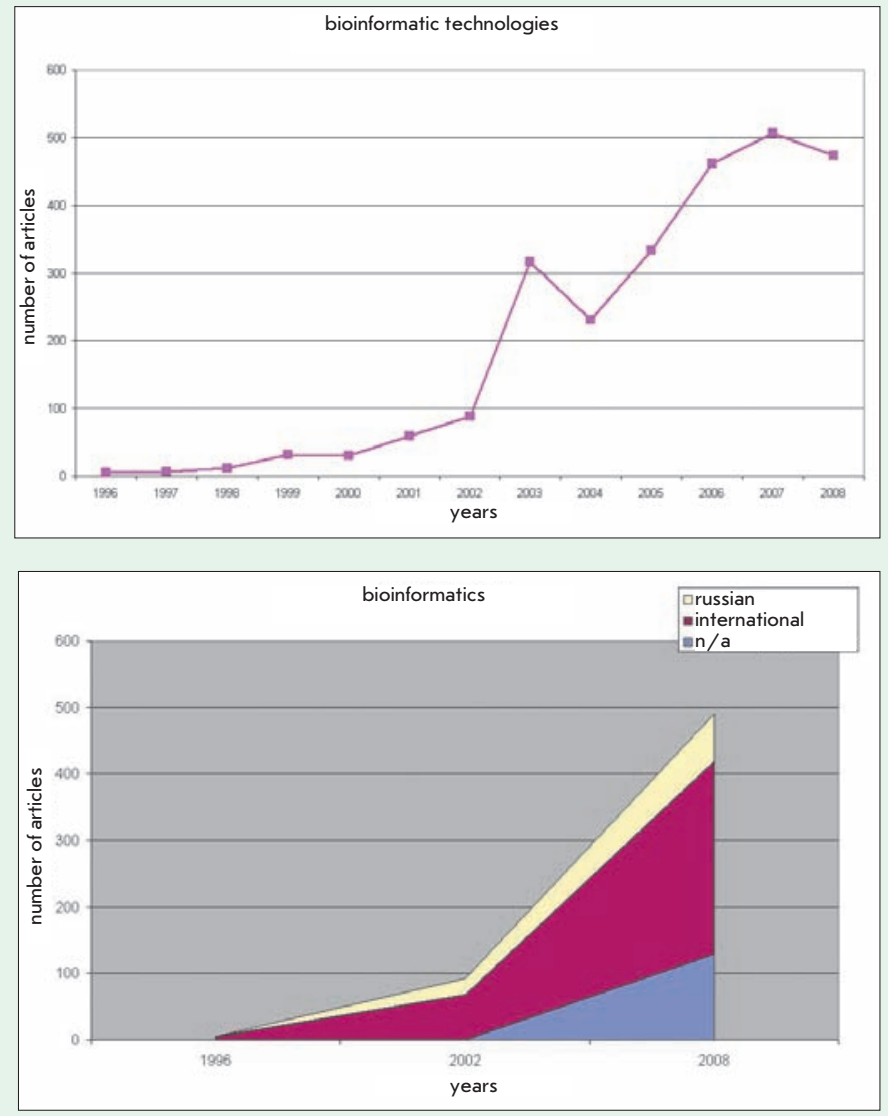

passed Switzerland in the number of publications (13,700 articles in 2008). In 12 years, the number of articles written by South Korean authors has increased 5-fold, from 3,300 to 16,700 articles.

Russia, on the other hand, after an insignificant increase in $\mathrm{PA}$ between 2000 and 2003 , is now the only country among the BRIC members to actually demonstrate a decrease in its PA (from 6,400 to 5,900 articles a year).

Let's consider in detail the characteristics of the publication activity of Russian authors for each of the spheres of the Living Systems referred to among the critical technologies.

\section{BIO-INFORMATION TECHNOLOGIES}

Bio-Information technologies are a relatively new branch within the life sciences characterized by intensive development in the whole world. The significant increase in the volume of investigations carried out in this field is related to the need for processing the large bulk of data generated during the course of conducting biological experiments.

Russian bioinformatics is no exception. Since the end of the 1990 s, when work in this field was rather rare, Russian scientists have increased their PA up to 500 articles a year. In spite of the fact that the last year was characterized by a decrease in the growth rate of publication activity, investigations in this sphere reflect rapid progress (Fig. 2). The key words used for this search were: bioinformatics, comparative genomics, and computational.

The projects in this field are carried out at the international level and are published largely in international English journals, such as Bioinformatics, Nucleic Acid Research, The Journal of Bioinformatics and Computational Biology, Molecular Phylogenetics and Evolution, The Journal of Molecular Biology, In Silico Biology, BMC Genomics, BMC Bioinformatics, and BMC Evolutionary Biology. As for
Russian journals, those which commonly publish articles on bio-information technology include: Biochemistry, Molecular Biology, Biophysics, Genet$i c s$, and Reports of the Russian Academy of Sciences.

According to SCOPUS data, in 2008, the average number of co-authors per published article in this field was 7 , and the average number of articles published by each collaborator was 3 . Therefore, the approximate number of researchers actively working in this field is 1,000 .

The following institutes of the Russian Academy of Sciences (RAS) stand out for a significant number of published articles on bioinformatics: The Institute of Cytology and Genetics, Siberian Branch (84 articles); The Engelhardt Institute of Molecular Biology (82); The Sobolev Institute of Mathematics, Siberian Branch (69); The Institute of Cytology (40); and The Kharkevich Institute for Information Transmission Problems (38).

The institutes of the Russian Academy of Medical Sciences (RAMS) rarely conduct research in this sphere, and most of those studies were conducted at the Orekhovich Institute of Biomedical Chemistry (37 studies); some investigations are being carried out in the Blokhin Russian Cancer Research Center (16 studies), the Gamalei Institute of Epidemiology and Microbiology (13 studies), and the Ivanovsky Institute of Virology (11 studies).

The State Scientific Research Institute for Genetics and the Selection of Industrial Microorganisms is unrivaled among the state scientific centers in terms of the number of articles on bio-information technology (102 articles), surpassing the Belozersky Institute of Physico-Chemical Biology, M.V. Lomonosov Moscow State University (42), and the Joint Institute for Nuclear Research in Dubna ( 27 articles).

The M.V. Lomonosov Moscow State University is the absolute leader among institutions of higher education in terms of the number of publications (448): The St. Petersburg State University (67), Novosibirsk State University (57), and St. Petersburg State Polytechnic University (33) are far behind. 


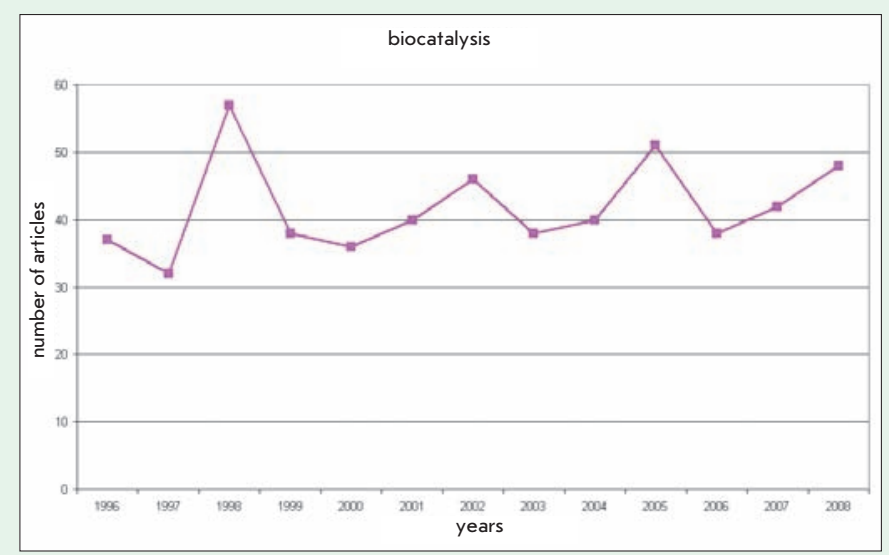

Fig. 4. Number of articles written by Russian researchers on the subject of biocatalytic technology.
Thus, today, the field of bio-information technology is actively developing. In the period between 2002 and 2008 , the number of publications increased almost 5-fold. Research articles in this field are at a very high level, with most of the articles being published in international journals (Fig. 3). This finding can be easily explained: research in bioinformatics does not require any expensive laboratory equipment or reagents. Computers and software are a major work instrument for bio-information scientists. The spheres of living systems studied on laboratory tables are rarely found in scientific periodicals.

\section{BIO-CATALYTIC, BIOSYNTHETIC, AND BIOSENSOR TECHNOLOGIES}

This category of critical technologies pertaining to living systems can be divided into three independent subcategories, each of which should be analyzed separately.

\section{BIO-CATALYTIC TECHNOLOGY}

Among the Russian Academies of Sciences, bio-catalytic technologies are pursued most actively in the RAS and RAMN institutes. However, the number of publications on this subject included in the SCOPUS database generally ranges between 40 and 50 articles a year, not considering the recent uptrend (Fig. 4). The key words used for this search were biocatalysis, enzymatic, and catalysis.

Investigations carried out by our scientists in this field are commonly published in such Russian journals as Biochemistry, Microbiology, Bioor- ganic Chemistry, and Russian Chemical Reviews. However, many articles are also published in such international journals as FEBS Letters, Biotechnology and Bioengineering, Biocatalysis and Biotransformation, and The Journal of Molecular Catalysis B Enzymatic.

According to the SCOPUS data, in 2008 , the average number of co-authors per published article was 5, and the average number of articles published by each collaborator was 1.2. Thus, the estimated number of researchers actively working in this field is 200 .

An analysis of those organizations whose research scientists work most actively in this field shows that, among the research institutes and state scientific centers, research in this field is most often carried out in the State Scientific Research Institute of Genetics and Selection of Industrial
Microorganisms (12 articles) and in the Gauze New Antibiotics Research Institute (8).

Among the RAS institutes, the ones with the highest PA activity are the Boreskov Institute of Catalysis, Siberian Branch (23 articles); the Zelinsky Institute of Organic Chemistry (19); and the Skryabin Institute of Biochemistry and Physiology of Microorganisms (12).

Among the RAMS institutes, the majority of these investigations are conducted at the Institute of Biomedical Chemistry (12 articles) and the AllRussian Cardiological Scientific Center (7).

Among the Institutions of Higher Education, the majority of the studies in question are carried out at M.V. Lomonosov Moscow State University (175 articles), while other institutions have a few publications to their name.

As a whole, the publication activity of Russian scientists in the field of bio-catalytic technology is rather low. However, it should be noted that $60 \%$ of all published articles in this field are published in international journals (Fig. 5), which is testament to the level of qualification of the scientists who are working in this field.

\section{BIOSYNTHETIC TECHNOLOGY}

Biosynthetic technologies are being developed much more rapidly, which is most likely due to the fact that they have been the subject of investigation for a longer period of time in our country. However, the number of publications (which exceeds the number of bio-catalytic related articles) has
Fig. 5. The Relationship between the number of Russian articles on biocatalytic technology published in Russian journals versus in international journals.

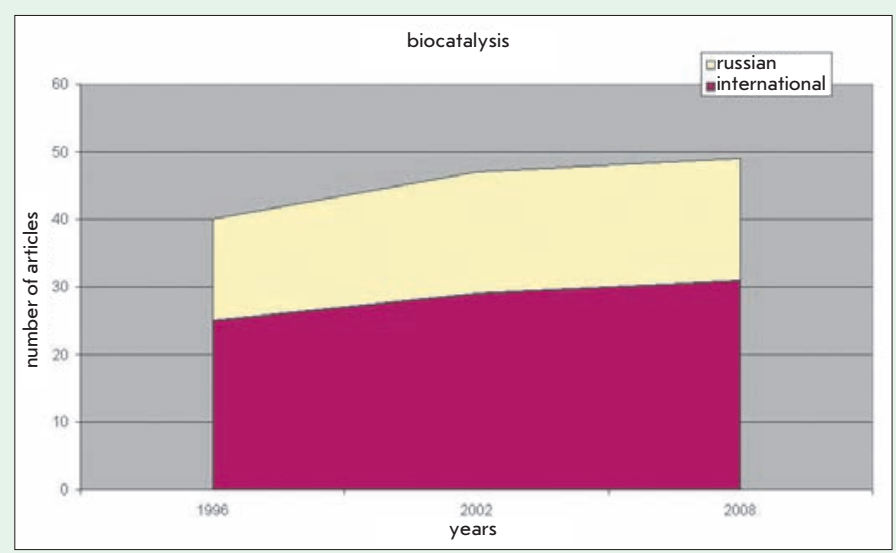


noticeably decreased over the last 3 years (Fig. 6). The key words used for this search were biosynthesis and synthesis.

The Investigations by Russian scientists in this field are mostly published in such Russian journals as Microbiology, Biochemistry, Bulletin of Experimental Biology and Medicine, Genetics, and Molecular Biology. Some of the studies are also published in such international journals as FEBS Letters, Pharmaceutical Chemistry Journal, the Journal of Biological Chemistry and Biophysics.

According to SCOPUS data, in 2008 the average number of co-authors cited in each published study was 5 , and the average number of articles published by each collaborator was 2.58 . Thus, the estimated number of researchers actively working in this field is around 700.

An analysis of those organizations whose research scientists are actively involved in this field shows that among the RAS institutes the highest PA belongs to the Skryabin Institute of Biochemistry and Physiology of Microorganisms (228 articles), followed by the Engelhardt Institute of Molecular Biology (206), the Zelinsky Institute of Organic Chemistry (178), the Timiryazev Institute of Plant Physiology (171), and the Institute of Molecular Genetics (109).

Among the RAMS institutes, the Gamalei Institute of Epidemiology and Microbiology (191 articles) is where investigations in this field are most often carried out.

Among the Institutions of Higher Education, research in biosynthetic technology was mainly carried out at M.V. Lomonosov Moscow State University (997 articles), followed by St. Petersburg State University (129), Sechenov Medical Academy of Moscow (90), Kazan State University (84), and the Moscow Lomonosov State Academy of Fine Chemical Technology (59).

Biosynthetic technology is, perhaps, the only one among the critical technologies that was characterized by such a dramatic decrease in PA during the period between 2002-2008, though in 1996-2002 some increase was noted. In spite of some increase in the number of publications in in-

Fig. 6. The number of articles written by Russian researchers in the field of biosynthetic technology.

Fig. 7. The Relationship between the number of Russian articles on biosynthetic technology published in Russian journals versus in international journals.

Fig. 8. The number of articles written by Russian researchers on the subject of biosensor technology.

ternational journals in 2008 (Fig. 7), the number of articles published in Russian journals in the same year decreased significantly.

\section{BIOSENSOR TECHNOLOGY}

Biosensor technology is a relatively new area of research within the living systems that is characterized by rapid development all over the world. In recent years, the number of Rus- sian publications in the area of biosensor technology included within the SCOPUS database has doubled, from 80 to 160 articles a year (Fig. 8). The key words used for this search were biosensor, biochip, and biomarker.

The research conducted by Russian scientists is mostly published in such international journals as Biosensors and Bioelectronics, The Journal of Analytical Chemistry, Bio-electrochemis- 
Fig. 9. The relationship between the number of Russian articles on the subject of biosensor technology published in Russian journals versus in international journals.

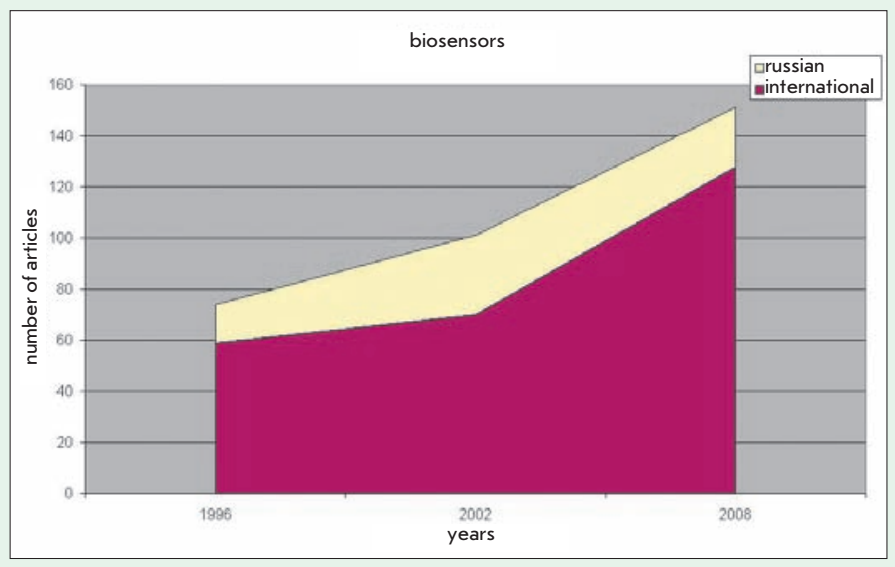

try, Analytical Letters, and Biomedical Engineering. Some of the studies are also published in Russian journals such as Applied Biochemistry and Microbiology and Molecular Biology.

According to SCOPUS data, in 2008 the average number of co-authors cited in each published study was 4.7, and the average number of articles published by each collaborator was 1.6. Thus, the estimated number of researchers actively working in this field is around 400 .

An analysis of those organizations whose research scientists are actively involved in this field shows that among the RAS institutes the highest PA is attributable to the Engelhardt Institute of Molecular Biology (49 articles), followed by the Prokhorov Institute of General Physics (33), the Shubnikov Institute of Crystallography (31), the Skryabin Institute of Biochemistry and Physiology of Microorganisms (17), and the Institute of Problems of Chemical Physics (15).

Among the RAMS institutes, research in this field is most often conducted at the Orekhovich Institute of Biomedical Chemistry (74 articles).

Among the Institutions of Higher Education, this research was carried out mainly at the M.V. Lomonosov Moscow State University (362 articles), Kazan State University (87) and St. Petersburg State University (65).

As this analysis suggests, biosensor technology is one of the fastest developing critical technologies. Between 2002 and 2008, the publication activity of those Russian scientists involved in research related to biosensor technology increased by $50 \%$, largely reflecting the increase in the number of publications in international journals (Fig. 9).

Hence, a general analysis of the critical technologies indicates that bi-

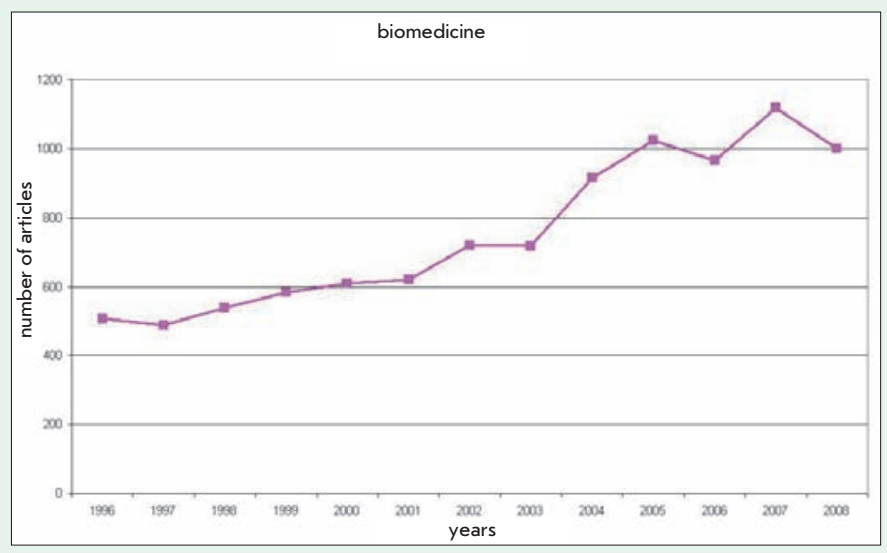

Fig. 10. The number of articles by Russian researchers in the field of biomedical technology related to human life support and defense. osensor technology is characterized by rapid development, whereas bio-catalytic technology remains somewhat stagnant, and biosynthetic technology actually suffered a setback, probably due to insufficient financial support.

\section{BIOMEDICAL AND VETERINARY TECHNOLOGIES RELATED TO HUMAN AND ANIMAL LIFE SUPPORT}

Biomedical and veterinary technologies were also analyzed separately, since the SCOPUS database can quite easily select the corresponding journals.

\section{BIOMEDICAL TECHNOLOGIES OF HUMAN LIFE SUPPORT AND DEFENSE}

The biomedical technologies pertaining to human life support have been the subject of investigation for a long time. In the last 12 years, the number of publications on the subject of biomedical technology has increased from 500 articles in 1996 to more than 1,000 in 2007. However, 2008 was marked by a decrease in the number of studies published (Fig. 10).

The articles by Russian scientists in this field were published mainly in Russian journals, such as Biomedical Chemistry, Biomedical Engineering, Medical Engineering, Bulletin of Experimental Biology and Medicine, Problems of Medical Chemistry, Medical Radiology, Genetics, Applied Biochemistry and Microbiology, etc. Some studies by Russian researchers were also published in such international journals as The Journal of Biological Chemistry, Human Physiology, Pharmaceutical Chemistry Journal, Biomedica Biochimica Acta, Critical Reviews in Biomedical Engineering, the Journal of Biomedical Optics, and many others.

According to the SCOPUS data, in 2008 the average number of coauthors cited in each published study was 5 , and the average number of articles published by each collaborator was 3.69. Thus, the estimated number of researchers actively working in this field is around 1,700 .

An analysis of those organizations whose research scientists are actively involved in this field shows that among the RAMS institutes the highest PA is attributable to the Orekhovich Institute of Biomedical Chemistry (711 arti- 
cles), followed by the All-Russian Cardiological Scientific Center (97), and the Blokhin Russian Cancer Research Center (97).

Among the RAS institutes, investigations in this field were carried out most frequently at the Institute of Biomedical Problems (471 articles), followed by the Prokhorov Institute of General Physics Institute (107), the Institute of Applied Physics (100), and the Semenov Institute of Chemical Physics (98).

Among the institutions of higher education, investigations were carried out mainly at the M.V. Lomonosov Moscow State University (1,282 articles), Sechenov Medical Academy of Moscow (166), St. Petersburg State University (159), and Russian State Medical University (136).

Among the scientific organizations conducting research in this field, the most active is the All-Russian Research Institute of Vitamins (118 articles), as well as the Vavilov State Optical Institute (39), the Joint Institute for Nuclear Research (38), and the Research and Practical Center of Medical Radiology (37).

As a whole, biomedical technologies are rapidly developing technologies within the living systems. In the period between 2002 and 2008, the number of articles published on the biomedical technologies increased by more than $30 \%$ and amounted to $70 \%$ of the total number of medical articles registered in the SCOPUS database. During that period, the number of articles published in international journals increased significantly (Fig. 11). This field of research is being actively pursued in the RAMS institutes, the profile RAS institutes, the institutes of the Ministry of Public Health and Social Development, and in medical schools.

\section{VETERINARY TECHNOLOGIES OF} ANIMAL LIFE SUPPORT AND DEFENSE

Veterinary technologies have been investigated in Russia for a long period of time as well. During the last 12 years, the number of publications in veterinary technologies included in the SCOPUS database has almost doubled, from 100 to 200 articles per year. However, the last few years have been characterized by a de-

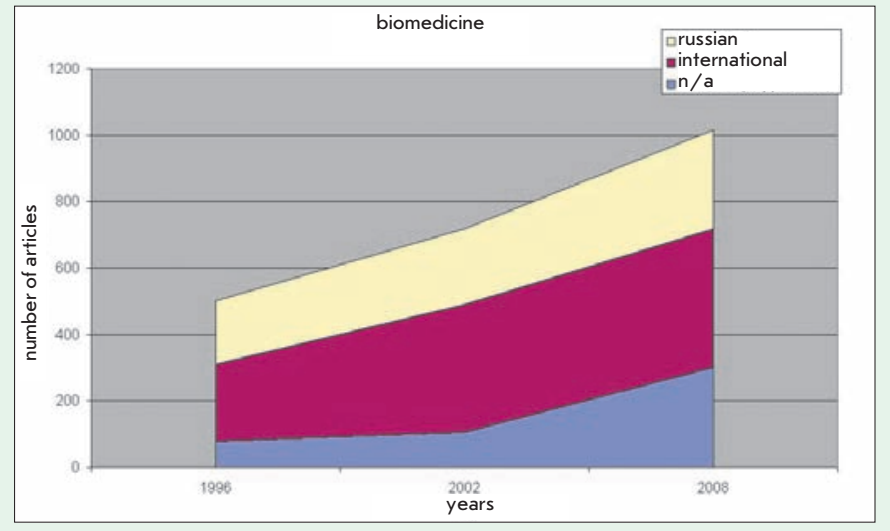

Fig. 11. Relationship between the number of Russian articles on biomedical technology of human life support and defense published in Russian journals versus in international journals.

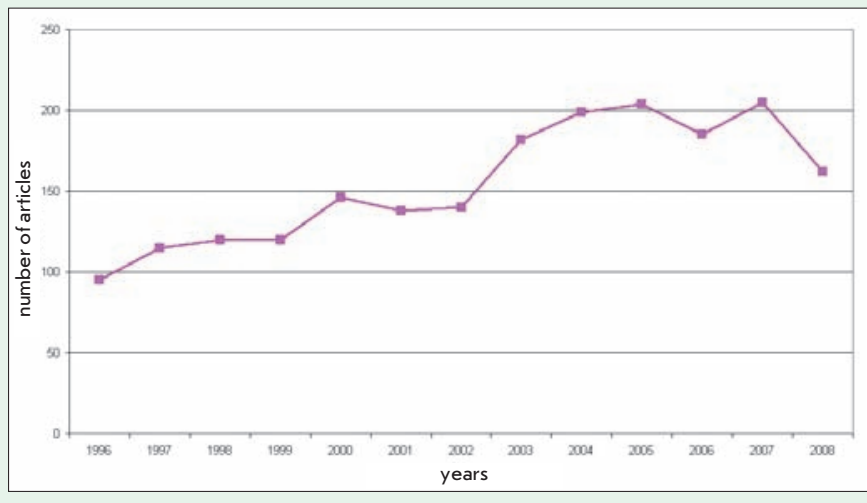

Fig. 12. The number of articles by Russian researchers in the sphere of veterinary technologies of animal life support and defense.

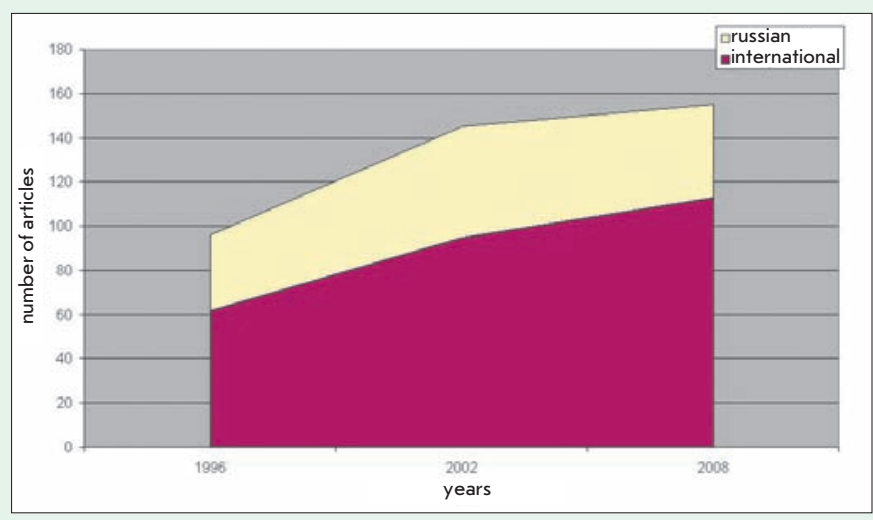

Fig. 13. Relationship between the number of Russian articles on veterinary technologies of animal life support and defense published in Russian and international journals. crease in the number of publications (Fig. 12).

Articles by Russian scientists in this field were published largely in Russian journals such as Radiobiology, Bulletin of Experimental Biology and Medicine, Radiation Biology and Radioecology, Biological Bulletin, Pharmacology and Toxicology, Journal of Microbiology, Epidemiology and Immunobiology, and Problems of Virology. Some investigations by Russian researchers in this field were also published in such international journals as Pharmaceutical Chemistry
Journal, Neuroscience and Behavioral Physiology, Vaccine, and the Journal of Biological Chemistry.

According to the SCOPUS data, in 2008 the average number of coauthors cited in each published study was 9 , and the average number of articles published by each collaborator was 1.56. Thus, the estimated number of researchers actively working in this field is approximately 1,200.

An analysis of those organizations whose researchers are involved in this field reveals that among the RAMS institutes, the highest PA is characteris- 
tic of the Medical Radiological Center (75 articles), as well as the Ivanovsky Institute of Virology (49), and the Gamalei Institute of Epidemiology and Microbiology (21).

Among research institutes and state scientific centers, investigations in this field were performed at the Institute of Biophysics of the Ministry of Public Health and Social Development, (M.p (89 articles), State Research Center of Virology and Biotechnology "Vector" (210), and Petrov Oncology Research Institute (21).

Among the RAS institutes, the highest PA in the considered sphere was noted at the Institute of Cytology (47 articles), Pavlov Institute of Physiology (45), Semenov Institute of Chemical Physics (38), Engelhardt Institute of Molecular Biology (36), and Institute of Biochemical Physics (35).

Among the institutions of higher education, investigations in this field were most often conducted at M.V. Lomonosov Moscow State University (261 articles), the Kirov Military Medical Academy (St. Petersburg, 44), and the Sechenov Medical Academy of Moscow (29).

\section{GENOMICS AND POST-GENOME DRUG FORMULATION}

Genomics and post-genome drug formulation represent a fairly new area of pharmacology, which promises to be very important in the future. In recent years, the number of publications included within the SCOPUS database has doubled from 300 to 600 articles per year in the field of genomics, and 100 to 200 articles in postgenomic pharmacology (Fig. 14). The key words used for this search were genomics, transcriptomics, proteomics, drug, target, drugs delivery, and antibiotic.

Articles by Russian scientists in the fields of genomics and post-genome drug formulation were published largely in Russian journals like Molecular Biology, Biochemistry, Microbiology, Bulletin of Experimental Biology and Medicine, Problems of Medical Chemistry, and Antibiotics and Chemotherapy. Some studies by Russian researchers were also published in such international journals as FEBS Letters, Pharmaceutical Chemistry Journal,

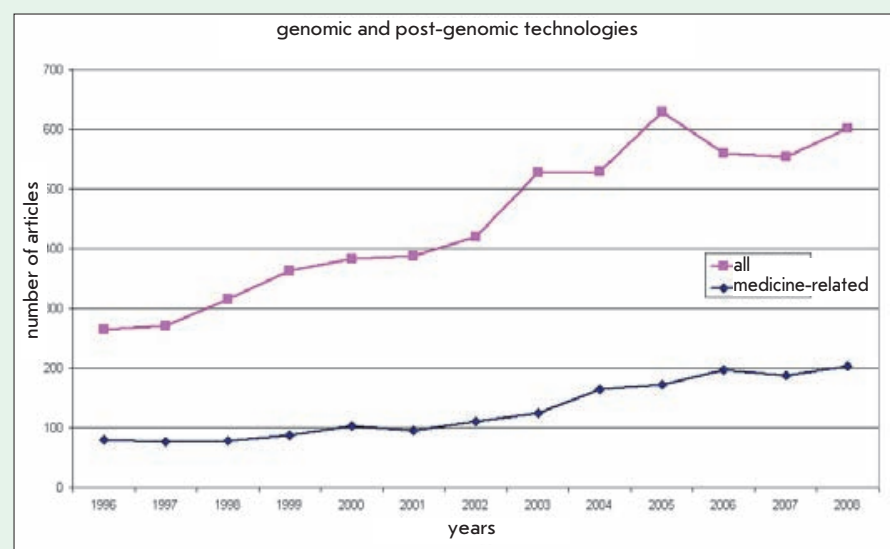

Fig. 14. The number of articles by Russian researchers in the sphere of genomics and post-genome drug formulation.

The Journal of Biological Chemistry and Biophysics.

According to the SCOPUS data, in 2008 the average number of coauthors cited in each published study was 7.15, and the average number of articles published by each collaborator was 3.24. Thus, the estimated number of researchers actively working in this field is around 400.

An analysis of those organizations whose research scientists are actively working in this field shows that among the RAS institutes the highest PA was generated by the Institute of Cytology (67 articles), and significant activity also took place at the Engelhardt Institute of Molecular Biology (66); the Institute of Cytology and Genetics, Siberian Branch (54); the Institute of Molecular Genetics (50); the Institute of Gene Biology (43); the Shemyakin and Ovchinnikov Institute of Bioorganic Chemistry (26); and the
Zelinsky Institute of Organic Chemistry (20).

Among the RAMS institutes, the majority of investigations in this field were conducted at the Blokhin Russian Cancer Research Center (46 articles), followed by the Ivanovsky Virology Institute (35), the Institute of Experimental Medicine (30), and the Orekhovich Institute of Biomedical Chemistry (21).

Among the research institutes and state scientific centers, studies in this area were carried out mostly at the State Scientific Research Institute of Genetics and Selection of Industrial Microorganisms (53 articles) and the State Research Center of Virology and Biotechnology Vector (22).

Among the Institutions of Higher Education, investigations in this area were most frequent at the M.V. Lomonosov Moscow State University (194 articles), while a little activity
Fig. 15. The Relationship between the number of Russian articles on genomics and post-genome pharmacology published in Russian journals versus in international journals.

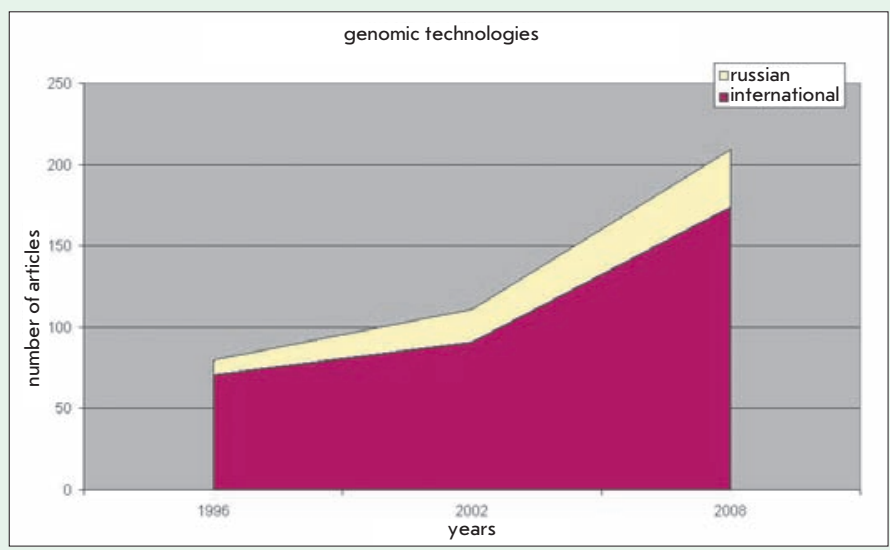


also came out of the St. Petersburg State University, and the Sechenov Medical Academy of Moscow (13).

Research into genomics and postgenome drug formulation is most actively pursued by scientists at the RAS, the RAMN, and the Ministry of Health institutes. Between 2002 and 2008, the number of publications in this field almost doubled. A significant majority of those articles found in the SCOPUS database were published in international journals (Fig. 15).

However, in spite of its relation to biomedicine, this critical technology is a medical component of the living systems which should be analyzed individually and in detail, as one of the factors that exert the greatest influence on the quality of life of the population.

Let's focus our attention on those areas of research that are developing rapidly throughout the world, such as specific drug delivery, the formulation of new medications to be used in the treatment of socially significant diseases, and the creation of new antibiotics and anti-cancer agents. In other words, let us focus on the major and most prominent areas of research.

\section{SYSTEMS OF SPECIFIC DRUG DELIVERY}

The creation of systems for the specific delivery of a particular drug to pathologically transformed cells and body tissues is one of the major directions of medical biotechnology development in the world. The development of these systems will make medications much more effective.

The Russian scientific organizations most actively involved in pursuing research on this field are the following:

RAS institutes: The Shemyakin and Ovchinnikov Institute of Bioorganic Chemistry (34 articles); the Institute of Chemical Biology and Fundamental Medicine, Siberian Branch (26); the Engelhardt Institute of Molecular Biology (82); and the Institute of Gene Biology (15). RAMS institutes: The Blokhin Russian Cancer Research Center (12 articles) and the Orekhovich Institute of Biomedical Chemistry (12).

The institutions of higher education: M.V. Lomonosov Moscow State University (31 articles), and Moscow Lomono-
Fig. 16. The number of articles published by Russian researchers in the field of specific drug delivery.
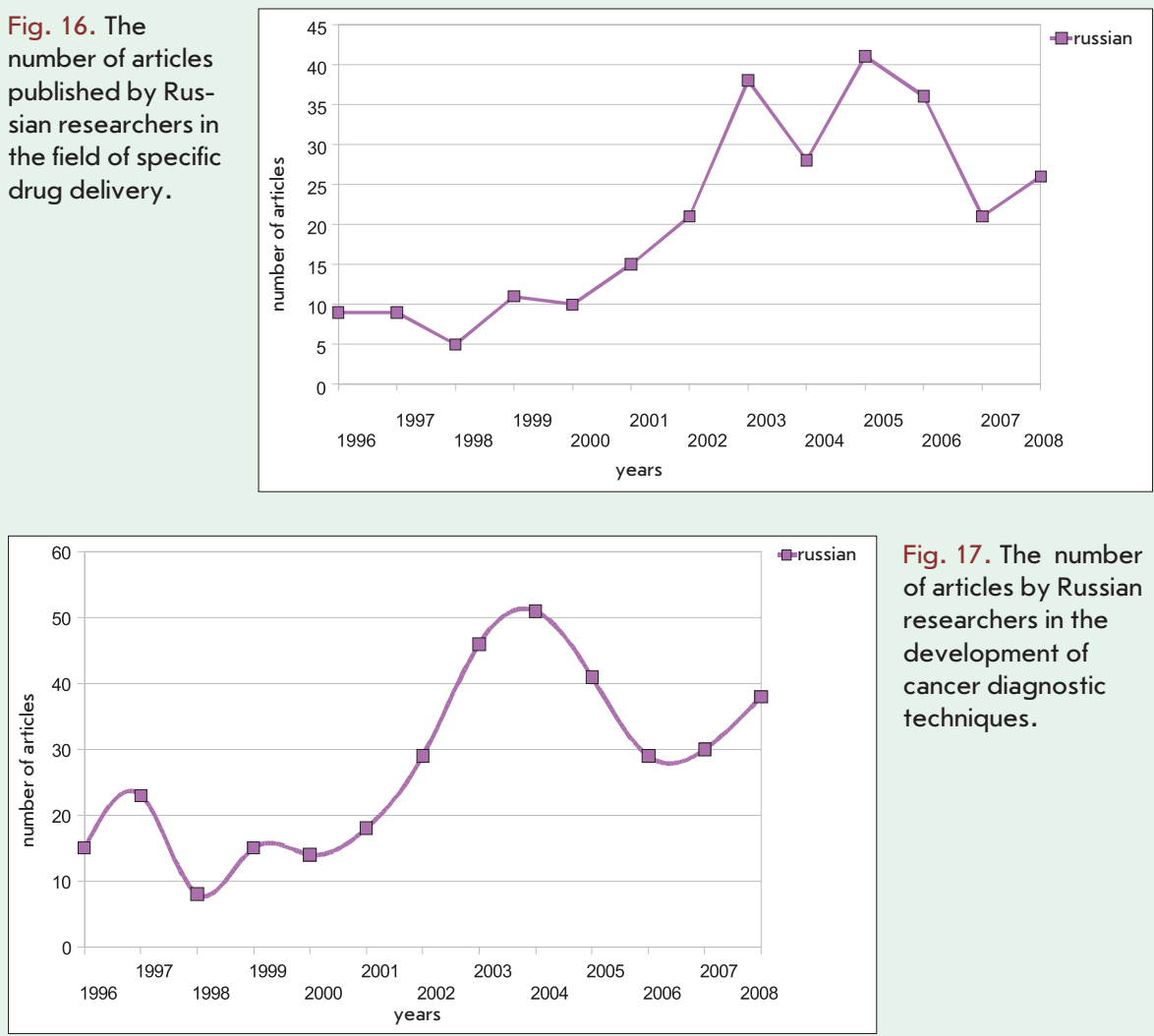

Fig. 17. The number of articles by Russian researchers in the development of cancer diagnostic techniques.

Fig. 18. The number of articles by Russian researchers in the area of new medications and techniques for the treatment of sociallysignificant diseases.

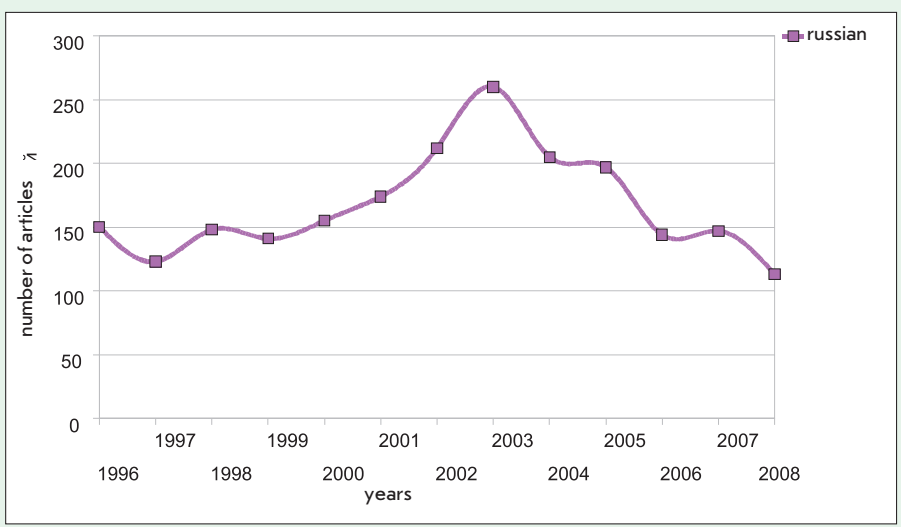

sov State Academy of Fine Chemical Technology (13).

Research institutes and federal science centers published only a handful of articles between them.

\section{DEVELOPMENT OF NEW ANTI- TUMOR DRUGS AND TECHNIQUES FOR CANCER DIAGNOSIS}

Among the RAS institutes, the theme was investigated the most actively by the Institute of Chemical Biology and Fundamental Medicine, Siberian
Branch (16 articles); Engelhardt Institute of Molecular Biology (13); Institute of Applied Physics (Nizhni Novgorod, 11); and Shemyakin and Ovchinnikov Institute of Bioorganic Chemistry (10).

Among RAMS institutes, investigations were carried out mainly at the Blokhin Russian Cancer Research Center (64 articles).

Development of New Medications and Treatment Modalities for Socially Significant Diseases. 
Fig. 19. The number of articles by Russian researchers in the field of cellular technology.

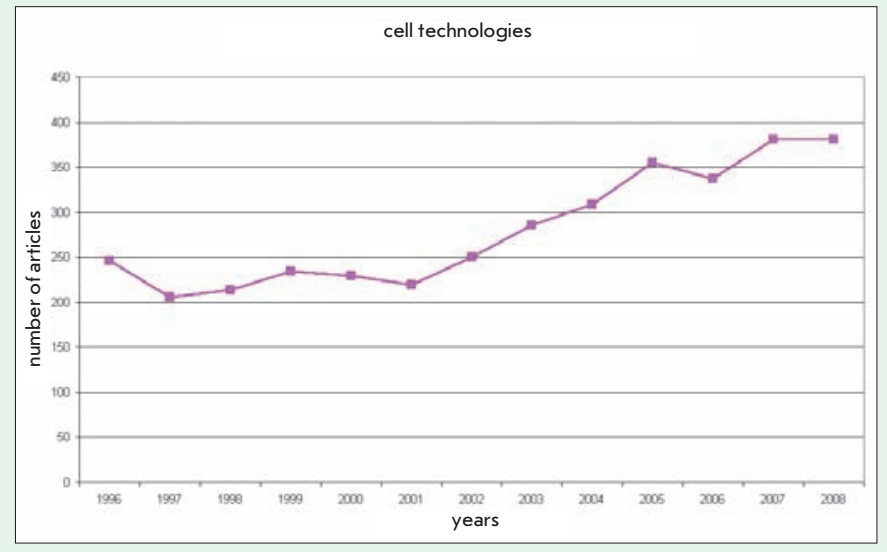

In this area, which is extremely important for our economy and our society due to the high prevalence and widespread distribution of socially significant diseases (for example, tuberculosis, AIDS, viral hepatitis C and B) the highest PA was observed at the following organizations:

RAS institutes: the Zelinsky Institute of Organic Chemistry (37 articles), the Nesmeyanov Institute of Organo-elemental Compounds (30), the Shemyakin and Ovchinnikov Institute of Bioorganic Chemistry (26), and the Elhardt Institute of Molecular Biology (25).

RAMS institutes: Zakusov Institute of Pharmacology (25 articles), Gauze New Antibiotics Research Institute (23), Institute of Biomedical Chemistry (22), and Orekhovich Institute of Biomedical Chemistry (19).

Institutions of higher education: M.V. Lomonosov Moscow State University (94 articles), Sechenov Medical
Academy of Moscow (60), and Moscow Lomonosov State Academy of Fine Chemical Technology (31).

\section{CELLULAR TECHNOLOGIES}

Cellular technologies are a relatively new area of research that has been developing quite successfully all over the world. In recent years, the number of publications in the field of cellular technologies included in the SCOPUS database has increased from 200 to almost 400 articles a year (Fig. 19). The key words used for this search were cell and technology.

The articles written by Russian scientists in the field of cellular technology were published, for the most part, in Russian journals such as The Bulletin of Experimental Biology and Medicine, Genetics, Molecular Biology, and Cytology. Some studies carried out by Russian researchers were also published in such international journals as The

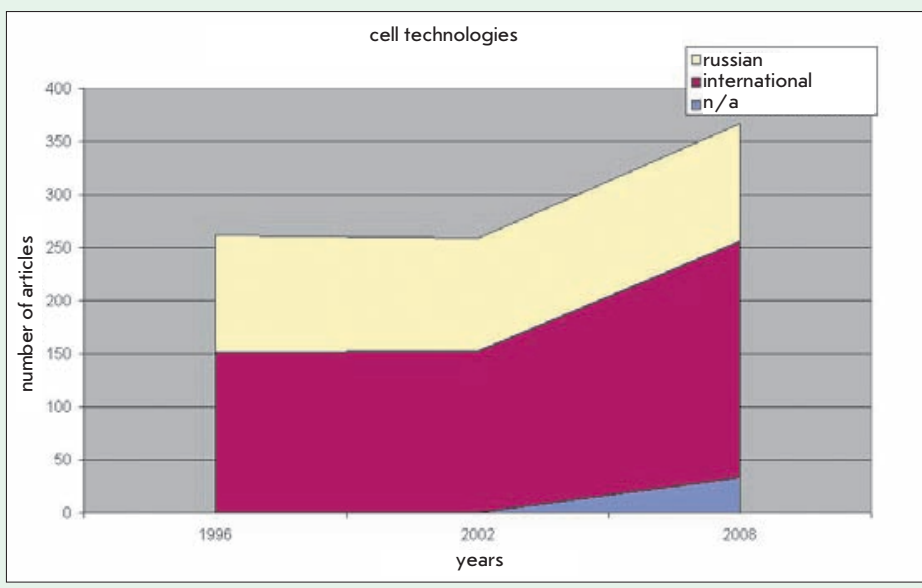

Fig. 20. The relationship between the number of Russian articles on the subject of cellular technologies published in Russian journals versus in international journals.
Pharmaceutical Chemistry Journal, the Proceedings of the National Academy of Sciences of the United States of America, FEBS Letters, the Journal of Biological Chemistry, and The Journal of Molecular Biology.

According to the SCOPUS data, in 2008 the average number of coauthors cited in each published study was 5.8, and the average number of articles published by each collaborator was 3.19. Thus, the estimated number of researchers actively working in this field is around 700 .

An analysis of those organizations whose research scientists are most actively involved in this field shows that among the RAS institutes, the Engelhardt Institute of Molecular Biology has the highest PA (99 articles), followed by the Skryabin Institute of Biochemistry and Physiology of Microorganisms (57), the Timiryazev Institute of Plant Physiology (51), the Institute of Gene Biology (50), the Institute of Molecular Genetics (47), the Institute of Cytology (43), and the Shemyakin and Ovchinnikov Institute of Bioorganic Chemistry (41).

Among the RAMS institutes, the majority of research studies in this field were conducted at the Orekhovich Institute of Biomedical Chemistry (56 articles), and somewhat less at the Blokhin Russian Cancer Research Center (46), and at the Institute of Experimental Medicine (29).

Among the institutions of higher education, these investigations were carried out mostly at M.V. Lomonosov Moscow State University (526 articles).

In spite of the fact that cellular technologies have been studied for a long time, they are still the fastest developing technologies within the living systems. In the period between 1996 and 2002, the publication activity of the scientists working in this field remained fairly at around 200 to 250 articles a year (almost half of which were published in international journals), but since 2002, the number of publications in this area has increased significantly, in large part due to the increased number of articles published in international journals (Fig. 20). Research into these technologies is conducted most frequently at RAS institutes and at institutions of higher education. 


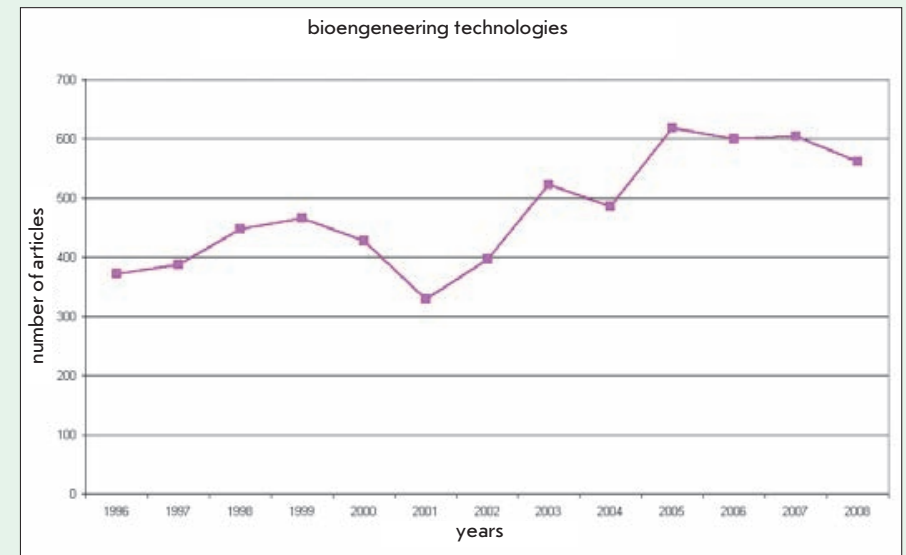

Fig. 21. The number of articles by Russian researchers in the field of bioengineering technologies.

\section{BIOENGINEERING TECHNOLOGY}

The bioengineering technologies represent a much older field of research, which has been extensively studied with significant success. The number of Russian publications on bioengineering technologies that are included in the SCOPUS database has increased by $50 \%$ (from less than 400 to almost 600 articles a year) (Fig. 21). The key words used for this search were bioengineering and tissue.

The articles by Russian scientists in the field of bioengineering technologies are published largely in such Russian journals as Biochemistry, Biophysics, Bulletin of Experimental Biology and Medicine, Applied Biochemistry and Microbiology, and Plant Physiology. However, a significant portion of these studies has also been published in such international journals as The Proceedings of the National Academy of Sciences of the United States of America, FEBS Letters, the Journal of Molecular Structure, Nucleic Acids Research, The Journal of Biological Chemistry, and Protein Engineering.

According to the SCOPUS data, in 2008 the average number of coauthors cited in each published study was 6.4 , and the average number of articles published by each collaborator was 2.05. Thus, the estimated number of researchers actively working in this field is around 1,900.

An analysis of those organizations whose research scientists are actively working in this field shows that among the RAS institutes, the Engelhardt Institute of Molecular Biology (256 arti- cles) has the highest PA, followed by the Institute of Molecular Genetics (138), the Shemyakin and Ovchinnikov Institute of Bioorganic Chemistry (73), and others.

Among the RAMS institutes, research in this field is most often conducted at the Orekhovich Institute of Biomedical Chemistry (74 articles), as well as at the All-Russian Cardiological Scientific Center (62), the Institute of Experimental Medicine (35), and the Blokhin Russian Cancer Research Center (31).

Among the research institutes and state scientific centers, studies in this realm are most often conducted at the State Scientific Research Institute of Genetics and Selection of Industrial Microorganisms (132 articles), and less so at both the Institute of Immunology of Federal Medico-Biologic Agency of Russia (43), and the Joint Institute for Nuclear Research (41).
Among the institutions of higher education, most studies in this field take place at M.V. Lomonosov Moscow State University (1,005 articles), and to a lesser degree also at St. Petersburg State University (122), and at the Moscow Institute of Physics and Engineering (102).

Bioengineering technologies have been successfully developed during the period analyzed (between 1996 and 2002). During that time, the increase in the number of articles published yearly was the result of publications in Russian journals, whereas in the years that followed (between 2002 and 2008), the number of studies printed in international journals has actually surpassed the number of those printed in Russian journals (Fig. 22).

\section{BIOCOMPATIBLE MATERIAL TECHNOLOGIES}

The biocompatible material technologies are another new and fast-developing field of research all over the world. The number of Russian publications on the subject of biocompatible material technologies that are included in the SCOPUS database has increased by 50\%, from 1 in 1996 to 25 in 2006 (Fig. 23). The key words used for this search were implant, tissue, and biocompatible.

The articles by Russian scientists on biocompatible material technologies have been published in such international journals as Biomaterials, Artificial Organs, Biosensors and Bioelectronics, and in Biomaterials, Artificial Cells and Artificial Organs.
Fig. 22. The relationship between the number of articles by Russian scientists on bioengineering technologies published in Russian journals versus in international journals.

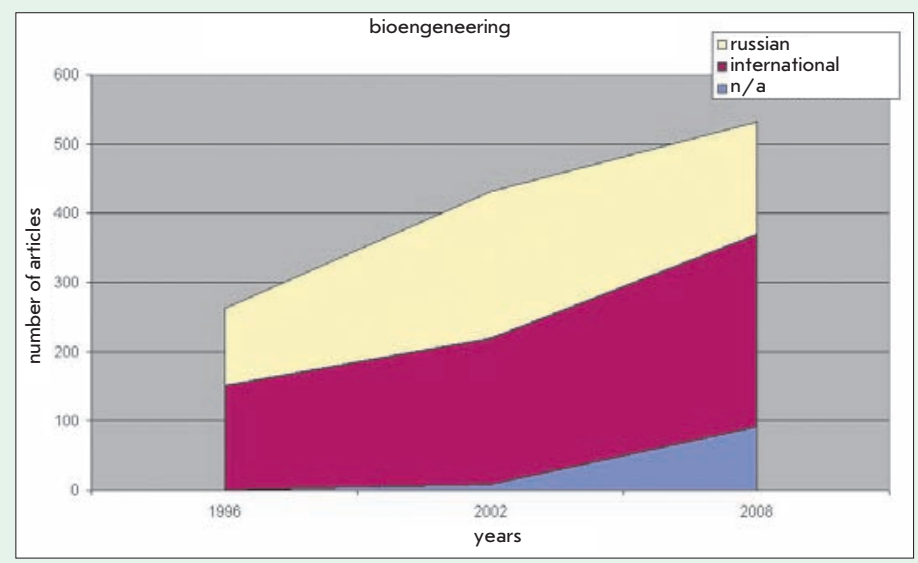


Fig. 23. The number of articles by Russian researchers in the field of biocompatible material technologies.

Fig. 24. The relationship between the number of articles by Russian scientists on biocompatible material technologies published in Russian journals versus in international journals.
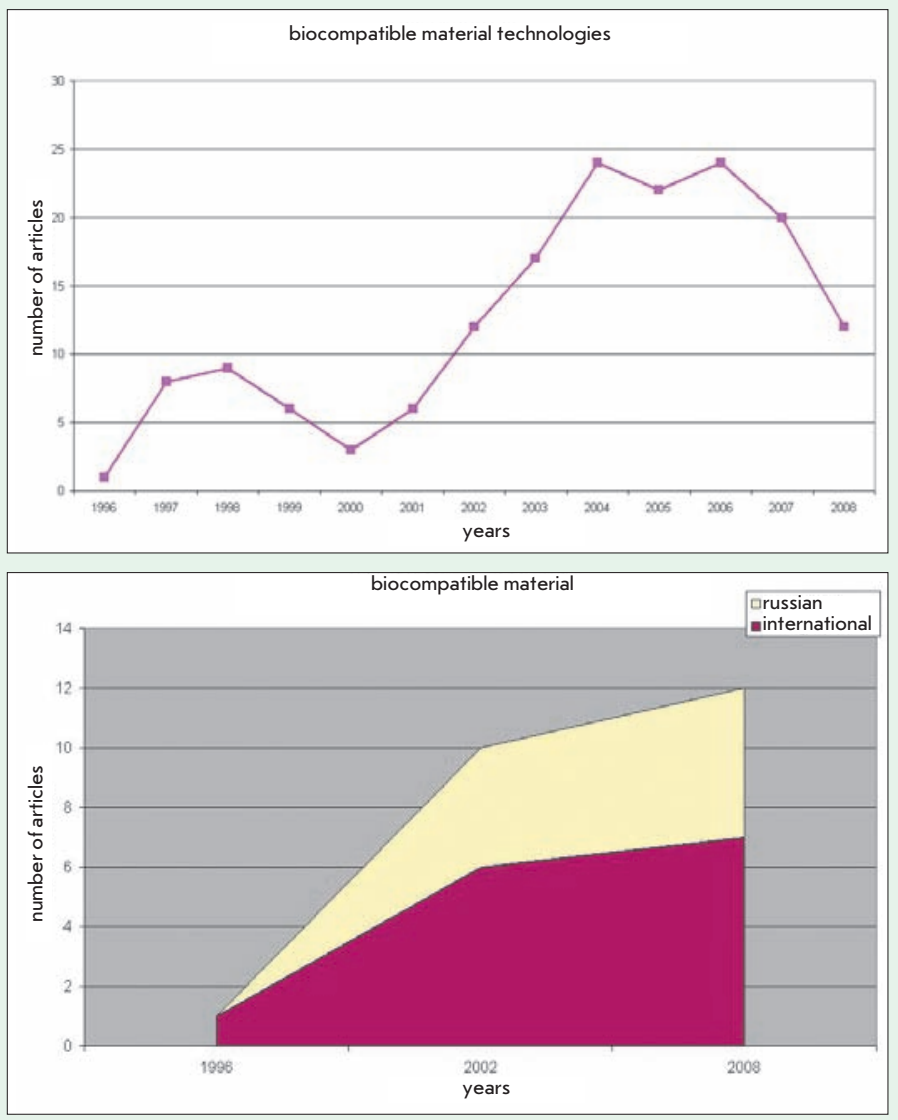

Fig. 25. An overview of the general publication activity of Russian scientists involved in investigations into the critical technologies referring to the sphere of living systems.

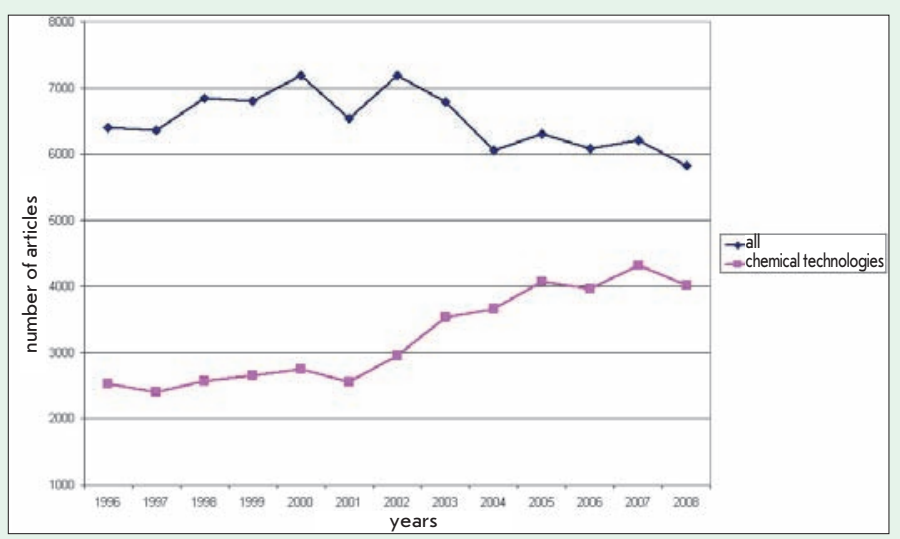

Some of the studies conducted by Russian researchers in this field have been published in such Russian journals as Medical Equipment, Bulletin of Experimental Biology and Medicine, Surgery, Archive of Pathology, and Problems of Medical Chemistry.

According to SCOPUS data, in 2008 the average number of co-authors cited in each published study was 4.83 , and the average number of articles published by each collaborator was
1. Thus, the estimated number of researchers actively working in this field is around 100 .

However, it should be noted that researchers at RAS and RAMS instisearch institutes have published only a few articles on biocompatible material technologies.

Among institutions of higher education, the majority of investigations in this field were conducted at M.V. Lotutes, state scientific centers, and re- monosov Moscow State University (24 articles).

As this analysis of the publication activity indicates, the degree of emphasis placed on biocompatible material technologies by the Russian scientific establishment is not entirely clear. On the one hand, during the period of time analyzed, those fields were the subject of an increased number of publications. On the other hand, the number of publications itself is insignificant, whereas the number of organizations carrying out research is significant enough. Moreover during the last 2 years, the number of publications has even decreased to some extent.

\section{THE LIVING SYSTEMS PRIORITY} DIRECTION AND OTHER BIOLOGICAL DISCIPLINES: REDISTRIBUTION OF ROLES

First, it should be noted that the adoption of both the Russian Federation's List of Critical Technologies and the Federal Target Program Research and Investigation of First-Priority Aspects of Russian Science and Technology had a significant influence on the development of living systems technologies. The number of publications in almost all categories of critical technologies increased. The number of articles on bioinformatics, biomedicine, biosensor and genome technologies accounts for two-thirds of the total number of Russian publications in the sphere of biology and medicine. In spite of the slight decrease in the total number of studies in the realm of biology and medicine, during the period from 2002 to 2008 , the number of articles related to critical technologies increased by one-third and in 2008 amounted to 4,000 articles per year. However, it ought to be noted that the publication activity in such critical areas as biocatalytic technologies and veterinary technologies of life support did not change, and publication activity in the sphere of biosynthetic technologies actually decreased.

It is worthy of note that the number of publications on critical technologies increased as a result of a redistribution of the total number of publications in the areas of biology and medicine in favor of critical technologies. At the same time, the decrease in the number of biological publications may be the 
result of a reduction in the number of researchers by 2008 , and may testify to the so-called over-saturation of researchers in this sphere (Fig. 26). At the same time, the increased publication activity of researchers working in the field of critical technologies of living systems is striking. As a whole, the total number of researchers actively working in this field is approximately 10,000 .

\section{THE IMPACT FACTORS (IF) OF RUSSIAN BIOLOGICAL JOURNALS}

The impact factor of journals is another indicator that reflects the number of articles published by Russian researchers. The impact factor (IF) is the number of quoted articles from a journal during the two previous years, divided by the total number of articles published in that same journal during those two years. In spite of some problems concerning the correct use of IFs, they are presently considered one of the formal criteria used for comparing investigations conducted between different disciplines. Thus, a change in the IF of Russian biological journals may be indicative of a change in the level of investigations, since this is where the results of those investigations are published.

12 Russian biological journals are registered in the SCOPUS database. Table 1 considers several variations of their IFs.

An increase in the Impact Factor is most notable for such journals as Molecular Biology, Biochemistry, and Bioorganic Chemistry (i.e., the journals which normally publish the articles of scientists from Russia's leading scientific organizations). During a 3-year period, the total impact factor of the 12 journals listed in Table 1 has increased from 4.303 to 6.076 .

\section{CONCLUSIONS}

The pattern of investigations observed allows us to make the following conclusions:

First, the lagging publication activity of Russian scientists in the realm of biology, which is now further behind even the developing countries, is striking. To call things as they are, Russia, compared to other developed scientific nations, is moving in the ex-

Table 1. Impact factors of Russian biological journals

\begin{tabular}{|c|c|c|c|c|}
\hline \multirow{2}{*}{ Name of journal } & \multicolumn{3}{|c|}{ Year } \\
\cline { 2 - 5 } Biochemistry & $\mathbf{2 0 0 4}$ & $\mathbf{2 0 0 5}$ & $\mathbf{2 0 0 6}$ & $\mathbf{2 0 0 7}$ \\
\hline Molecular Biology & 1.058 & 0.858 & 1.368 & 1.476 \\
\hline Bioorganic Chemistry & 0.623 & 0.435 & 0.33 & 0.805 \\
\hline Microbiology & 0.358 & 0.571 & 0.572 & 0.63 \\
\hline Plant Physiology & 0.539 & 0.534 & 0.543 & 0.597 \\
\hline Biophysics & 0.381 & 0.310 & 0.444 & 0.51 \\
\hline Biochemistry and Microbiology & 0.16 & 0.277 & 0.321 & 0.439 \\
\hline Journal of Higher Nervous Activity & 0.367 & 0.362 & 0.435 & 0.43 \\
\hline Gulletin of Experimental Biology and Medicine & 0.254 & 0.238 & 0.19 & 0.249 \\
\hline Journal of Evolutionary Biochemistry and Physiology & 0.178 & 0.238 & 0.206 & 0.199 \\
\hline Rews. Biological Series & 0.037 & 0.027 & 0.048 & 0.098 \\
\hline
\end{tabular}

act opposite direction. This process is likely a reflection of the more general trends occurring in Russian science, namely, the ageing of old cadres, and as a result, a decrease in the number of highly qualified researchers.

Second, we can observe some increase in the number of publications concerning the critical technologies as they relate to the living systems. It is quite possible that this is due to the adoption, in 2002, of the Federal Target Program Research and Investigation of First-Priority Aspects of Russian Science and Technology, as well as several programs from the Russian Academy of Sciences (Fundamental Sciences for Medicine, Molecular and Cell Biology). The increase in the IFs of the leading biological journals testifies to the improved quality of the investigators, whose research is being published by these journals.

Our analysis of the publication activity also shows that the research institutes and the institutions of higher learning working within the framework of the living systems remain segregated in pools, which points to the fact that investigations of the living systems in Russia still have some potential. The state should support and encourage this potential.

The next step in this analysis would naturally be an evaluation of the publication activity generated by different laboratories. This seems only logical, since it is only at the level of individual laboratories that the efficiency of scientific teams may be adequately analyzed. Furthermore, we are planning to examine the joint publication efforts of those Russian scientists working within the Russian scientific establishments and their colleagues in foreign countries, since these joint efforts provide a clear insight into the level and the quality of scientific work.

Finally, the last step involves an individual look at some of those scientists who are leaders in their respective fields, by evaluating the parameters of their publication activity (first of all, we are talking about the citation index). 